\title{
Numerical Study on the Characteristics of Air Flows through Helmet Bicycle Racing using the Effect of Variation of Trailing Edge
}

\author{
Syamsuri ${ }^{1, \text { a) }}$ Hasan Syafik Maulana ${ }^{1, b)}$ Achmad Syarifuddin ${ }^{1, c)}$ \\ ${ }^{1}$ Department of Mechanical Engineering, Institut Teknologi Adhi Tama Surabaya, Arief Rahman Hakim 100, \\ Surabaya, 60117 \\ e-mail: syamsuri@itats.ac.id
}

\begin{abstract}
Research on aerodynamics on racing bicycles always develops from time to time. The various geometry of a time-trial helmet produces different characteristics of fluid flow, this is due to the relative movements of air that are in the area throughout the body shape of the helmet. Basically, the fluid flow that passes on a racing bicycle helmet will produce a drag force, where this must be minimized to hinder the pace of drivers to achieve maximum speed, so drivers should pay attention to how to design the geometry of helmet that should be used. The computational fluid dynamics (CFD) method is used to simulate the case studies in this research. In this study, four kinds of models trailing edge geometry was varied to determine where the most optimal in accepting the drag force. The validation was also conducted to determine the suitability of this study with prior research, wherein this validation the results of this study are compared with the research owned (Sims and Jenkins, 2011). The results of this validation show that the resulting drag coefficient has a very small difference of 0.001 . The four models are simulated with Reynold number values of $7.14 \times 10^{4}, 1.00 \times 10^{5}$, and $1.16 \times 10^{5}$. The results of this study indicate that using differences in the geometry of the trailing edge affect the drag force that occurs. From the research result when Reynold $7.0 \times 10^{\wedge} 4$, the drag force produced by model 3 is bigger than model 1 and 2 which is equal to $0.182 \mathrm{~N}$. Whereas on Reynold which is bigger $1.16 \times 10 \wedge 5$ model 3 receives drag smaller than model 1 and 2 which is equal to $0.283 \mathrm{~N}$. In the world of bicycle racing, the difference in the small drag force affects the speed of the bicycle and affects the resulting victory.
\end{abstract}

Keywords: helmet bicycle; trailing edge; racing; Reynold number; CFD

\section{INTRODUCTION}

Helmets are a form of body protection worn on the head and are usually made of metal or hard materials such as kevlar, resin fiber, plastic, or other composite materials [1]. The racing bike type of helmet is designed slightly differently from other types of helmets in general, especially on the aerodynamics and dimensions weighs. Basically, the aerodynamic characteristics that occur in the body of a racing bicycle helmet are caused by the relative movement of the air along with the shape of the helmet body. Thus, around the helmet area a streamlined airflow pattern will be formed. Streamline at the farthest distance on the helmet will form a pattern that is parallel and undisturbed, while in the area around the helmet will have a slightly complex air pattern as a result of disruption of airflow 
from the design of the helmet. This results in the formation of a drag force which hinders the driver's speed to obtain maximum speed.

Analysis of the aerodynamics of racing bicycle helmets has been carried out by several researchers. Among the influences driving position resulting in a degree of helmet position also changes. In this case the helmet or driving position has a very high role in contributing to the drag force that occurs, which is about more than $65 \%$ of the total drag force that occurs, while other factors only contribute a small part [2-6]. The effect of ventilation from the helmet has been analyzed by Alam et al., In this study several models of racing helmets with various forms of ventilation were analyzed, and showed that the drag force received did not show a significant difference [7]. While the influence of the length cut trailing edge variation on the helmet time trial was also examined by Huda, et al., In his research showing that $75 \mathrm{~mm}$ cuts on the trilling edge can produce a decrease in drag force that occurs [8]. Previous researchers have not discussed modifications to the trailing edge. The drag coefficient is greatly affected by the turbulence of the flow near the trailing edge. Modifications were made to minimize flow turbulence near the trailing edge especially when the velocity was increased.

In this study variations in the shape of the trailing edge on the helmet time trial were analyzed using the CFD method to determine the trend of flow and drag coefficient resulting from this variation. This is intended to obtain the appropriate geometry to produce the maximum speed of the application in the field.

\section{Research Method}

To obtain research data that is according to the intended purpose, the shape of the trailing edge is varied by 3 types, as shown in Figure 1, the model is then analyzed using the computational fluid dynamic (CFD) method. In this study the Reynold number is also varied to show the results obtained from various conditions, where the Reynold number used is $7.14 \times 10^{4}, 1.00 \times 10^{5}$, and $1.16 \times 10^{5}$. The air density used in this study is $1 \mathrm{~kg} / \mathrm{m}^{3}$ and its viscosity $1.81 \times 10^{-5} \mathrm{~Pa}$. $\mathrm{s}$ with temperature $293.2^{\circ} \mathrm{K}$ at atmospheric pressure. The parameters are then processed in numerical software using the finite volume method. In this study validation was also conducted to determine the suitability of this study with prior research, wherein this validation the results of this study are compared with the research owned [9]. The results of this validation show that the resulting drag coefficient has a very small difference of 0.001 where in the Sims and Jenkins study the resulting drag coefficient was 0.590 while the resulting drag coefficient was 0.589 , so that this comparison indicated that the parameters used in this study were already corresponding.

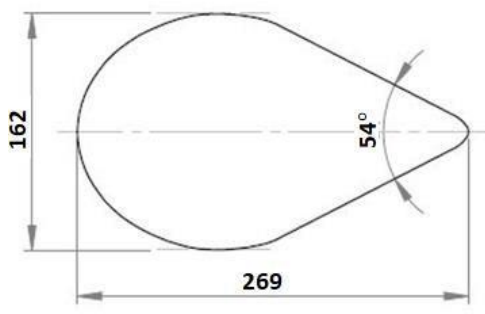

PRODUCTION HELMET

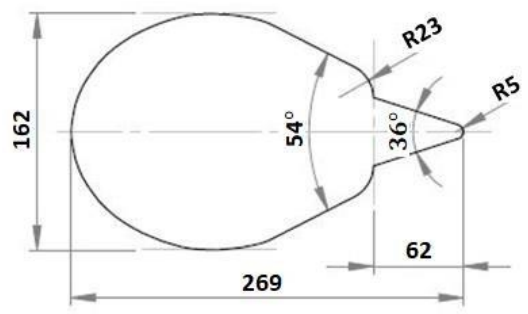

MODEL 1

Figure 1. Trailing Edge Variation Models 


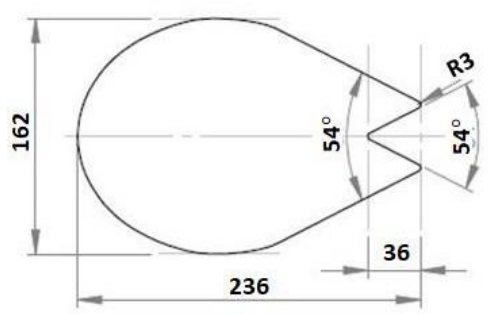

MODEL 2

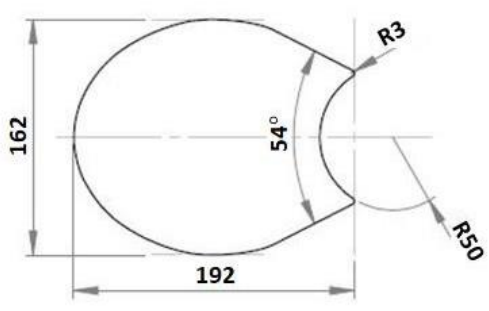

MODEL 3

Figure 1. Trailing Edge Variation Models (continued)

\section{RESULt AND Discussions}

\section{Flow Visualization of the Effect of the Variation of Tailing Edge}

In this study, four models were compared to determine the difference in drag force received on each variation of the trailing edge model. With the parameters mentioned above, the four models show quite different visualization results as shown in Figure 2 . In Figure 2.a shows a trace of fluid flow when crossing the body production helmet with a Reynold number $1.00 \times 10^{5}$ and it can be seen that the flow will move to follow the contour of the body. However, when the fluid will cross the trailing edge, oscillating Karman vortex is formed on the wake area behind the helmet body. This vortex is a phenomenon where fluid flow at the back of a time-trial helmet forms a repetitive vortex pattern caused by unsteady flow separation around blunt bodies. This alternating flow pattern forms on the back of the helmet body and then moves down on the edge of the trailing edge, causing drag on the helmet body. The wake area behind the body is formed when the fluid flow begins to dissolve. Wake is a low-pressure area caused by a change in momentum due to flow separation. And generally wake can be found in the area behind the body. From the characteristics of this fluid flow that will affect the size of the drag force received by the body. In model 1 with a variation of the trailing edge shown in Figure 2.b, the phenomenon or characteristic of fluid flow that occurs is not much different from the characteristics or contours of the flow that occurs in $2 \mathrm{D}$ time-trial helmet production.

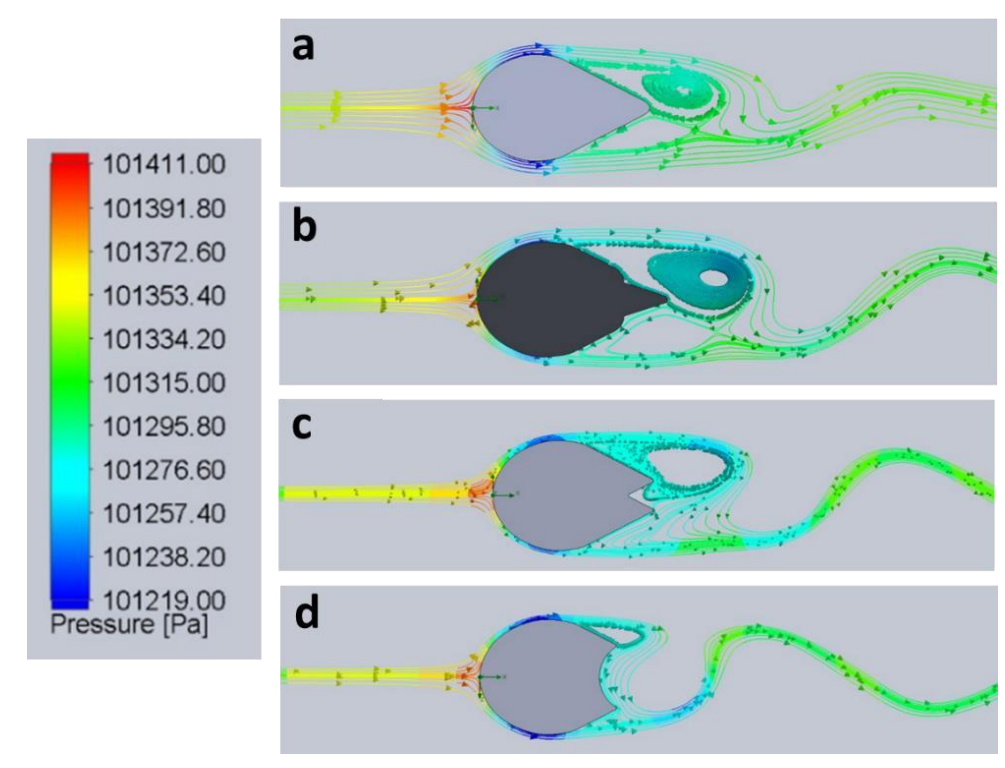

Figure 2. Flow Visualization at Reynolds Numbers $1.00 \times 10^{5}$ (a) Productions Helmet, (b) Model 1, (c) Model 2, (d) Model 3 
In model 2 with a variation of the trailing edge shape as shown in Figure 2.c., whirl flow pattern is formed in the rear area of the helmet body and then down to the edge of the trailing edge, causing a drag on the body of a helmet. However, if you look closely, the size of the vortex formed on the trailing edge model 2 modification is slightly smaller than the previous model. Therefore, the pressure drop that occurs on the trailing edge is not as large as the pressure drop that occurs on trailing edge model 1 modification and helmet timetrial production, this results in a decrease in the drag force that occurs with a time-trial helmet body with trailing edge model modifications 2 . In the modification of trailing edge model 3 a vortex formation is formed that is much smaller and faster to escape as shown in Figure 2.d., it is shown that by using the trailing edge models 3 , the drag force received will be smaller than the drag force that is received by the other models in this study.

\section{The Effect of Trailing Edge Variations on Drag and Drag Coefficients}

In this section, the simulation results that have been completed are then stored and retrieved data in the form of a drag $(\mathrm{Fd})$ that occurs on each model of production helmet, modified trailing edge model 1 , modified trailing edge model 2 , and on trailing edge model 3 modification. All simulation results of the models obtained by meshing condition, $\mathrm{k}-\varepsilon$ turbulence models, domain and boundary and at the same solver settings, which results are shown in Table 1. In the table shows the comparison of the drag force to the Reynolds number on various trailing edge variations studied in this study. In general, the higher the Reynolds number, the higher the drag force that occurs. This is because the Reynolds number is a function of speed. By doing variations on the trailing edge body resembling a helmet it can reduce the impact of the drag force that occurs. In Reynolds number $7.14 \times$ $10^{4}$ the smallest drag force occurs in the time-trial helmet model 2. But when the Reynolds number reaches $1.00 \times 10^{5}$ and $1.16 \times 10^{5}$ the smallest drag force occurs in the timetrial helmet model 3 . This corresponds to flow visualization that occurs in each model studied. In models 2 and 3 , it can be seen that traces of flow characteristics result in smaller vortices compared to model 1 and on the production helmet, resulting in a drag force value that is smaller than the model 1 or production helmet

Table 1. Comparison of drag force on various models and Reynold numbers

\begin{tabular}{cccc}
\hline \multirow{2}{*}{ Model } & \multicolumn{3}{c}{ Drag Force (N) } \\
\cline { 2 - 4 } & $\mathbf{7 . 1 4 \times 1 0 ^ { 4 }}$ & $\mathbf{1 . 0 0 \times 1 0 ^ { 5 }}$ & $\mathbf{1 . 1 6 \times 1 0 ^ { 5 }}$ \\
\hline Production helmet & 0.175 & 0.299 & 0.357 \\
Model 1 & 0.173 & 0.305 & 0.362 \\
Model 2 & 0.168 & 0.291 & 0.320 \\
Model 3 & 0.182 & 0.278 & 0.283 \\
\hline
\end{tabular}

Table 2. Comparison of drag coefficient on various models and Reynold numbers

\begin{tabular}{cccc}
\hline \multirow{2}{*}{ Model } & \multicolumn{3}{c}{ Drag Coefficient (Cd) } \\
\cline { 2 - 4 } & $\mathbf{7 . 1 4 \times 1 0 ^ { 4 }}$ & $\mathbf{1 . 0 0 \times 1 0 ^ { 5 }}$ & $\mathbf{1 . 1 6 \times 1 0 ^ { 5 }}$ \\
\hline Production helmet & 0.675 & 0.589 & 0.521 \\
Model 1 & 0.666 & 0.599 & 0.529 \\
Model 2 & 0.647 & 0.572 & 0.467 \\
Model 3 & 0.702 & 0.547 & 0.412 \\
\hline
\end{tabular}

Data in the form of the drag force (Fd) obtained from the simulation results are then used to calculate the value of the coefficient of drag (Cd) by using the formula as used in the study of (Syamsuri, et al., 2018), which results are shown in Table 2. In general, the higher the Reynolds number, the lower the drag coefficient value that occurs. This is because the Reynolds bill is a function of speed as shown in the graph of Figure 3. By doing variations on the trailing edge body resembling a helmet it can reduce the impact of the drag coefficient that occurs. In Reynolds number $7.14 \times 10^{4}$ the smallest drag coefficient 
occurs in the time-trial helmet model 2. But when the Reynolds number reaches $1.00 \times$ $10^{5}$ and $1.16 \times 10^{5}$ the smallest drag coefficient occurs in the time-trial helmet model 3 .

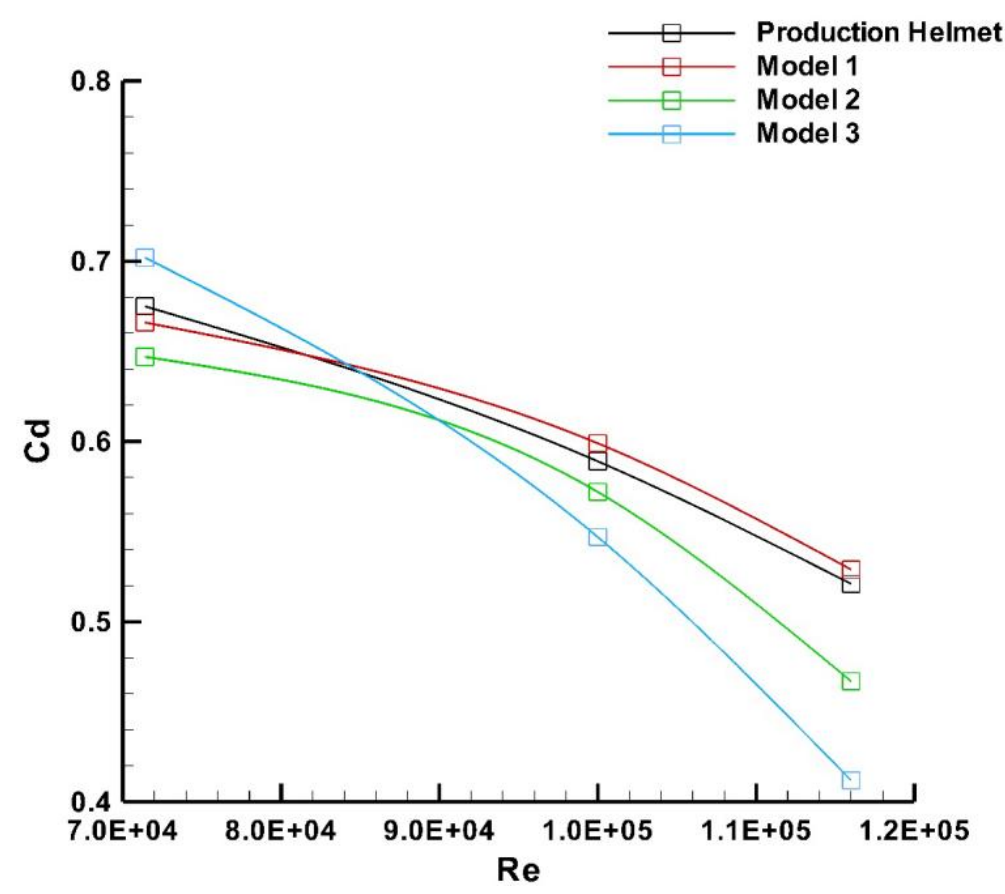

Figure 3. Graph of comparison of drag coefficient (Cd) to Reynolds number (Re) on various variations of Trailing Edge

\section{Conclusions}

The results of validation with previous studies have shown that there is a good agreement between this study and previous researchers. In general, the difference in error is about $0.1 \%$. The smallest drag force is obtained in model 3 of $0.278 \mathrm{~N}$ in the Reynolds number $1.00 \times 10^{5}$ and $0.283 \mathrm{~N}$ at the Reynolds number $1.16 \times 10^{5}$. Whereas in the Reynolds number $7.14 \times 10^{4}$ the smallest drag force is obtained in model 2 of 0.168 .

\section{ACKNOWLEDGMENTS}

Thanks to Institut Teknologi Adhi Tama Surabaya for supporting and funding this research. The scheme of this research is to use an independent research scheme.

\section{REFERENCES}

1. D. G. Fitzpatrick, M. Goh, D. C. Howlett, and M. Williams, International Journal of Oral and Maxillofacial Surgery 47 (9), 1121 (2018).

2. F. Beaumont, R. Taiar, G. Polidori, H. Trenchard, and F. Grappe, Journal of Biomechanics 67, 1 (2018).

3. Bert Blocken, Thijs Defraeye, Erwin Koninckx, Jan Carmeliet, and Peter Hespel, Computers \& Fluids 71, 435 (2013).

4. Thijs Defraeye, Bert Blocken, Erwin Koninckx, Peter Hespel, and Jan Carmeliet, Journal of Biomechanics 43 (7), 1262 (2010).

5. Thijs Defraeye, Bert Blocken, Erwin Koninckx, Peter Hespel, and Jan Carmeliet, Journal of Biomechanics 44 (9), 1695 (2011).

6. Syamsuri, M Hasan Syafik, and Yudho Putro Iswanto, MATEC Web Conf. 204, 06001 (2018).

7. Firoz Alam, Harun Chowdhury, Ho Zhi Wei, Israt Mustary, and Gary Zimmer, Procedia Engineering 72, 691 (2014). 
JEMMME (Journal of Energy, Mechanical, Material, and Manufacturing Engineering)

Vol. 6, No. 1, 2021 doi: $10.22219 / \mathrm{jemmme.v6i1.12573}$

8. Khoiri Huda, Eky Novianarenti, and Syamsuri Syamsuri, VANOS Journal of Mechanical Engineering Education (Vol 3, No 1 (2018)) (2018).

9. Bradford W. Sims and Peter E. Jenkins, ASME 2011 International Mechanical Engineering Congress and Exposition (54921), 453 (2011). 